\title{
Separability and hidden symmetries of Kerr-Taub-NUT spacetime in Kaluza-Klein theory
}

\author{
Göksel Daylan Esmer \\ Istanbul University, Department of Physics, Vezneciler, 34134 Istanbul, Turkey
}

(Dated: October 16, 2018)

\begin{abstract}
The Kerr-Taub-NUT spacetime in the Kaluza-Klein theory represents a localized stationary and axisymmetric object in four dimensions from the Kaluza-Klein viewpoint. That is, it harbors companion electromagnetic and dilaton fields, thereby showing up the signature of the extra fifth dimension. We explore the separability structure of this spacetime and show that the Hamilton-Jacobi equation for geodesics admits the complete separation of variables only for massless geodesics. This implies the existence of the hidden symmetries in the spacetime, which are generated by the conformal Killing tensor. Using a simple trick built up on a conformally related metric (an "effective" metric) with the Killing tensor, we construct the explicit expression for the conformal Killing tensor.
\end{abstract}




\section{INTRODUCTION}

It is long known that the Einstein vacuum field equations admit an intriguing exact solution that describes a localized stationary and axisymmetric object, the so-called rotating gravitational dyon. In other words, general relativity (GR) admits a simple generalization of the ordinary Kerr spacetime to include the "magnetic mass", in addition to the ordinary mass [1] (see also Refs. [2, 3]). This spacetime is known as the Kerr-Taub-NUT (NewmanUnti-Tamburino) solution of GR. The presence of the magnetic mass or the NUT charge in the spacetime results in conical singularities on its axis of symmetry, thereby making the spacetime asymptotically nonflat. This in turn enables one to elaborate on the gravitomagnetic analogue of Dirac's string quantization condition [3]. Thus, one can think of the NUT charge as "dual" to the ordinary mass in GR just as the electric charge can be thought of as dual to the magnetic charge in the theory of electromagnetism. Continuing this line of the interpretation, one can also consider the conical singularities as the physical source of a string which lies on the axis of symmetry of the black hole spacetime [4]. On the other hand, the conical singularities can be removed by imposing a periodicity condition on the time coordinate, but it creates another pathology in the spacetime, namely, the appearance of closed timelike curves (CTCs). Thus, in general, it is hard to interpret the Kerr-TaubNUT spacetime as describing a regular rotating black hole, in contrast to the original Kerr spacetime.

In addition to these undesired features, the Kerr-Taub-NUT spacetime also possesses a number of remarkable properties. Among them, the separability property is of particular interest [5-7]. It turns out this spacetime shares the hidden symmetries of the ordinary Kerr spacetime, generated by a second-rank Killing tensor [8], thereby rendering both the Hamilton-Jacobi and Klein-Gordon equations completely integrable. Due to these properties, the Kerr-Taub-NUT spacetime has been an attractive model for exploring various gravitomagnetic phenomena in asymptotically nonflat spacetimes (see [6, 7, 9, 10] and references therein). It is important to note that the spacetimes with the NUT charge turn out to be an inseparable part of the low-energy string theory as well. Here one can show that the duality symmetries of the effective action allow to construct new stationary TaubNUT type solutions to the theory [11, 12]. The NUT charge also provides a kind of new electric-magnetic duality that was revealed in supersymmetric configurations of the Kerr- 
Newman-Taub-NUT-AdS spacetime [13]. The spacetimes with NUT charges have also been studied in higher-dimensional GR with a cosmological constant, where the most general Kerr-AdS solution with multi-NUT charges was found in [14]. It was shown that this spacetime admits a closed conformal Killing-Yano 2-form which encodes all its hidden symmetries [15, 16].

The investigation of the Kerr-Taub-NUT spacetime is also of interest from the KaluzaKlein viewpoint. In [17], it was shown that the Kerr-Taub-NUT solution in the Kaluza-Klein theory carries the signature of the extra fifth dimension, acquiring the electric and dilaton charges though the dilaton charge is not an independent parameter. This solution was obtained by employing a well-known solution generating procedure [18, 19] that in essence involves uplifting the original Kerr-Taub-NUT metric to five dimensions and boosting it in the flat fifth dimension with a subsequent Kaluza-Klein reduction to four dimensions. Clearly, the resulting metric satisfies the coupled Einstein-Maxwell dilaton field equations in four dimensions.

Meanwhile, such a procedure of the construction of the solution raises a natural question: Are the separability property and the associated hidden symmetries of the original spacetime preserved by the solution generating procedure? In a recent work [20], this question was explored for the Kerr-Kaluza-Klein black hole. In this Letter, we continue this line of the exploration and address the same question for Kerr-Taub-NUT spacetime in the Kaluza-Klein theory. In Section 2 we briefly discuss the spacetime metric and show that the HamiltonJacobi equation for geodesics in the spacetime under consideration exhibits the complete separability structure only for massless geodesics. Clearly, it is the conformal Killing tensor that generates the hidden symmetries underlying the separability in the massless case. In Section 3 we employ a simple trick, which involves a conformally related metric with the pertaining Killing tensor, and we give the explicit expression for the conformal Killing tensor.

\section{THE METRIC AND THE HAMILTON-JACOBI EQUATION}

We recall that the Kerr-Taub-NUT spacetime in the Kaluza-Klein theory represents a localized rotating object with a NUT charge in four dimensions from the Kaluza-Klein viewpoint and involves the Maxwell and dilaton fields. The details of the construction of 
the spacetime metric can be found in [17]. Here we will only focus on some basic steps of the construction. The first step amounts to uplifting the original Kerr-Taub-NUT solution [1] to five dimensions, by adding to it a flat spacelike dimension. This results in the fivedimensional metric which in the Boyer-Lindquist coordinates is given by

$$
d s_{5}^{2}=-\frac{\Delta}{\Sigma}(d t-\chi d \phi)^{2}+\Sigma\left(\frac{d r^{2}}{\Delta}+d \theta^{2}\right)+\frac{\sin ^{2} \theta}{\Sigma}\left[a d t-\left(r^{2}+a^{2}+\ell^{2}\right) d \phi\right]^{2}+d y^{2},
$$

where the metric functions

$$
\begin{aligned}
& \Delta=r^{2}-2 M r+a^{2}-\ell^{2}, \quad \Sigma=r^{2}+(\ell+a \cos \theta)^{2}, \\
& \chi=a \sin ^{2} \theta-2 \ell \cos \theta,
\end{aligned}
$$

and $M$ is the mass parameter, $a$ is the rotation parameter, $\ell$ is the NUT charge. At the second step, one needs to boost this metric in the fifth dimension, applying the transformation

$$
\begin{aligned}
& t \rightarrow t \cosh \alpha+y \sinh \alpha \\
& y \rightarrow y \cosh \alpha+t \sinh \alpha
\end{aligned}
$$

with the boost velocity $v=\tanh \alpha$. Putting now the resulting metric into the standard Kaluza-Klein form

$$
d s_{5}^{2}=e^{-2 \Phi / \sqrt{3}} d s_{4}^{2}+e^{4 \Phi / \sqrt{3}}(d y+2 A)^{2},
$$

and performing a compactification along the fifth dimension, one obtains the four-dimensional metric

$$
\begin{aligned}
d s_{4}^{2}= & -\frac{1}{B} \frac{\Delta}{\Sigma}(d t-\chi \cosh \alpha d \phi)^{2}+B \Sigma\left(\frac{d r^{2}}{\Delta}+d \theta^{2}\right)-\frac{\Delta \sin ^{2} \theta}{B} \sinh ^{2} \alpha d \phi^{2} \\
& +\frac{\sin ^{2} \theta}{B \Sigma}\left[a d t-\left(r^{2}+a^{2}+\ell^{2}\right) \cosh \alpha d \phi\right]^{2},
\end{aligned}
$$

which is accompanied by the potential one-form $A$ and by the dilaton field $\Phi$. We have

$$
A=\frac{Z \sinh \alpha}{2 B^{2}}\left[\cosh \alpha d t-\left(\chi+\frac{2 \ell \cos \theta}{Z}\right) d \phi\right], \quad \Phi=\frac{\sqrt{3}}{2} \ln B
$$

where

$$
B=\left(1+Z \sinh ^{2} \alpha\right)^{1 / 2}, \quad Z=2 \frac{M r+\ell(\ell+a \cos \theta)}{\Sigma} .
$$


It is straightforward to check that this solution satisfies the equation of motion derived from the four-dimensional action of the Kaluza-Klein theory

$$
S=\int d^{4} x \sqrt{-g}\left[R-2(\partial \Phi)^{2}-e^{2 \sqrt{3} \Phi} F^{2}\right]
$$

where $F=d A$. Meanwhile, the physical parameters of the metric; the total mass, angular momentum and the total electric charge are given by

$$
\mathcal{M}=\frac{M}{2}\left(\frac{2-v^{2}}{1-v^{2}}\right), \quad J=\frac{a M}{\sqrt{1-v^{2}}}, \quad Q=\frac{M v}{1-v^{2}} .
$$

As for the dilaton charge, it is not independent as one can express it in terms of the other parameters (see Refs. [17, 18] for details). Thus, the spacetime metric in (5) generalizes the Kerr-Taub-NUT solution of GR to include the effects of the extra dimension that show up through the appearance of the electric and dilaton charges in the spacetime. For $\ell=0$, this solution goes over into the boosted Kerr black hole metric that was earlier found in [19].

Let us now consider the Hamilton-Jacobi equation for geodesics in the background of spacetime (5). It is given by

$$
\frac{\partial S}{\partial \lambda}+\frac{1}{2} g^{\mu \nu} \frac{\partial S}{\partial x^{\mu}} \frac{\partial S}{\partial x^{\nu}}=0
$$

where $\lambda$ is an affine parameter. Using the fact the spacetime under consideration admits two commuting timelike and spacelike Killing vectors one can assume that

$$
S=\frac{1}{2} m^{2} \lambda-E t+L \phi+F(r, \theta),
$$

where $F(r, \theta)$ is an arbitrary function. The constants of motion are the mass $m$, the total energy $E$ and the angular momentum $L$ of the particle. Substituting this action together with the contravariant components of metric (5), given by

$$
\begin{aligned}
& g^{00}=\frac{1}{B \Sigma}\left[\Sigma \sinh ^{2} \alpha+\frac{\chi^{2} \cosh ^{2} \alpha}{\sin ^{2} \theta}-\frac{\left(r^{2}+a^{2}+\ell^{2}\right)^{2} \cosh ^{2} \alpha}{\Delta}\right], \\
& g^{11}=\frac{\Delta}{B \Sigma}, \quad g^{22}=\frac{1}{B \Sigma}, \quad g^{03}=\frac{\cosh \alpha}{B \Sigma}\left[\frac{\chi}{\sin ^{2} \theta}-\frac{a}{\Delta}\left(r^{2}+a^{2}+\ell^{2}\right)\right], \\
& g^{33}=\frac{1}{B \Sigma}\left(\frac{1}{\sin ^{2} \theta}-\frac{a^{2}}{\Delta}\right)
\end{aligned}
$$

into equation (10), we reduce it into the form

$$
\begin{aligned}
& \Delta\left(\frac{\partial F}{\partial r}\right)^{2}+\left(\frac{\partial F}{\partial \theta}\right)^{2}+\left[\Sigma \sinh ^{2} \alpha+\left(\frac{\chi^{2}}{\sin ^{2} \theta}-\frac{\left(r^{2}+a^{2}+\ell^{2}\right)^{2}}{\Delta}\right) \cosh ^{2} \alpha\right] E^{2} \\
& +\left(\frac{1}{\sin ^{2} \theta}-\frac{a^{2}}{\Delta}\right) L^{2}-2 \cosh \alpha\left[\frac{\chi}{\sin ^{2} \theta}-\frac{a}{\Delta}\left(r^{2}+a^{2}+\ell^{2}\right)\right] E L=-m^{2} B \Sigma
\end{aligned}
$$


With equation (17) in mind, it is not difficult to see that separation of the $r$ and $\theta$ variables in this equation does not occur due to the presence of the function $B$ on the right-hand side. Meanwhile, such a separation does occur for massless geodesics $(m=0)$. As is known, this fact of separability guarantees the existence of a new conserved quantity along the null geodesics. It is the second-rank symmetric conformal Killing tensor that generates the hidden symmetries underlying the separability in the massless case [8]. In what follows, we explore the hidden symmetries and construct the explicit form for the conformal Killing tensor.

\section{THE CONFORMAL KILLING TENSOR}

In a recent work [20], the conformal Killing tensor and the associated hidden symmetries of the Kerr-Kaluza-Klein black hole were explored by using a simple trick based on a conformally related metric with the Killing tensor. Here we shall use similar trick for constructing the conformal Killing tensor of the the Kerr-Taub-NUT spacetime in the Kaluza-Klein theory. As it was shown above, the associated spacetime metric does not allow the full separation of variables for the massive Hamilton-Jacobi equation. That is, the spacetime does not admit the Killing tensor $K_{\mu \nu}$ given by the equation

$$
\nabla_{(\lambda} K_{\mu \nu)}=0
$$

where $\nabla$ denotes the covariant differentiation with respect to the spacetime metric $g_{\mu \nu}$. On the other hand, one can assume that such a Killing tensor exists for a conformally related metric $h_{\mu \nu}$ (an effective metric) given as

$$
h_{\mu \nu}=e^{2 \Omega} g_{\mu \nu}
$$

where $\Omega$ is a scalar function and $g_{\mu \nu}$ is supposed to be the original metric in (5). It is not difficult to show that the associated covariant derivatives of a second-rank symmetric tensor $P_{\mu \nu}$ are related by

$$
\left.D_{(\lambda} P_{\mu \nu)}=\nabla_{(\lambda} P_{\mu \nu)}-4 P_{(\mu \nu} \Omega_{, \lambda)}-g_{(\mu \nu} I_{\lambda}\right)
$$

where

$$
I_{\lambda}=-2 g^{\alpha \tau} P_{\lambda \tau} \Omega, \alpha
$$


The operator $D$ denotes covariant differentiation with respect to the metric $h_{\mu \nu}$ and the comma stands for the partial derivative. Next, it is straightforward to show that with the tensor $P_{\mu \nu}$ given in the form

$$
P_{\mu \nu}=e^{-4 \Omega} K_{\mu \nu}
$$

where $K_{\mu \nu}$ is the Killing tensor for the effective metric $h_{\mu \nu}$, equation (16) can be cast to the defining equation for the conformal Killing tensor. That is, we have

$$
\left.\nabla_{(\lambda} P_{\mu \nu)}=g_{(\mu \nu} I_{\lambda}\right)
$$

On the other hand, taking the scalar function $\Omega$ as

$$
\Omega=-\frac{1}{2} \ln B
$$

one can show that the massive Hamilton-Jacobi equation in the background of the effective metric $h_{\mu \nu}$ admits the complete separation of variables. In this case, the factor $B$ on the right-hand side of equation (13) disappears and for the action $S$ in the form

$$
S=\frac{1}{2} m^{2} \lambda-E t+L \phi+S_{r}(r)+S_{\theta}(\theta)
$$

we obtain two independent ordinary differential equations given by

$$
\begin{aligned}
& \Delta\left(\frac{d S_{r}}{d r}\right)^{2}-\frac{1}{\Delta}\left[\left(r^{2}+a^{2}+\ell^{2}\right) \cosh \alpha E-a L\right]^{2}+r^{2}\left(m^{2}+\sinh ^{2} \alpha E^{2}\right)=-K, \\
& \left(\frac{d S_{\theta}}{d \theta}\right)^{2}+\frac{1}{\sin ^{2} \theta}(\chi \cosh \theta E-L)^{2}+(\ell+a \cos \theta)^{2}\left(m^{2}+\sinh ^{2} \alpha E^{2}\right)=K,
\end{aligned}
$$

where $K$ is a constant of separation. It is clear that the separability implies the existence of a new quadratic integral of motion $K=K^{\mu \nu} p_{\mu} p_{\nu}$. This is associated with the hidden symmetries of the effective metric $h_{\mu \nu}$, which are generated by the irreducible Killing tensor $K^{\mu \nu}$. Taking this into account in equation (23), with the normalization condition $m^{2}=$ $-h^{\mu \nu} p_{\mu} p_{\nu}$ in mind, we find the explicit form for the Killing tensor. We have

$$
\begin{aligned}
& K^{\mu \nu}=\delta_{\theta}^{\mu} \delta_{\theta}^{\nu}+\left[(\ell+a \cos \theta)^{2} \sinh ^{2} \alpha+\frac{\chi^{2}}{\sin ^{2} \theta} \cosh ^{2} \alpha\right] \delta_{t}^{\mu} \delta_{t}^{\nu}+\frac{1}{\sin ^{2} \theta} \delta_{\phi}^{\mu} \delta_{\phi}^{\nu} \\
& +\left(\delta_{t}^{\mu} \delta_{\phi}^{\nu}+\delta_{\phi}^{\mu} \delta_{t}^{\nu}\right) \frac{\chi}{\sin ^{2} \theta} \cosh \alpha-h^{\mu \nu}(\ell+a \cos \theta)^{2} .
\end{aligned}
$$

For the vanishing boost parameter $\alpha=0$, this expression reduces to the Killing tensor for the original Kerr-Taub-NUT spacetime (see, for instance, [21]). 
Next, we need to calculate the nonvanishing components of the "current vector" in (17). Using equations (15) and (18), with equation (20) in mind, it is not difficult to show that the current one-form is given by

$$
\begin{aligned}
I= & \frac{\sinh ^{2} \alpha}{B \Sigma^{2}}\left\{(\ell+a \cos \theta)^{2}\left[M\left(2 r^{2}-\Sigma\right)+2 r \ell(\ell+a \cos \theta)\right] d r\right. \\
& \left.+a r^{2} \sin \theta\left[\left(\Sigma-2 r^{2}\right) \ell+2 M r(\ell+a \cos \theta)\right] d \theta\right\} .
\end{aligned}
$$

Here we have also used the $K_{r}^{r}$ and $K_{\theta}^{\theta}$ components of the Killing tensor in (24). It is interesting to note that this expression does not vanish for $a=0$. In other words, even the Schwarzschild-Taub-NUT spacetime fails to retain its Killing tensor in the Kaluza-Klein framework. Thus, the hidden symmetries of the original Kerr-Taub-NUT spacetime, generated by the irreducible Killing tensor, fail to survive from the Kaluza-Klein viewpoint. Meanwhile, the hidden symmetries generated by the conformal Killing tensor do exist, guaranteeing the complete separability of variables in the Hamilton-Jacobi equation for massless geodesics. With (20) in mind, it is straightforward to verify that the conformal Killing tensor in equation (18), in which the Killing tensor is obtained by lowering (with respect to the effective metric $h_{\mu \nu}$ ) the contravariant indices of the tensor in (24), satisfies equation (19) with the current vector given in (25).

\section{CONCLUSION}

The purpose of this Letter was to address the question of how and to what extent the hidden symmetries of the original Kerr-Taub-NUT spacetime survive in the Kaluza-Klein framework. Exploring the separability structure of the Hamilton-Jacobi equation in the Kerr-Taub-NUT-Kaluza-Klein spacetime, we have found that the complete separation of variables occurs only for massless geodesics. That is, the spacetime admits the hidden symmetries generated by the conformal Killing tensor. Using a simple trick built up on the conformally related metric with the Killing tensor, we have constructed the explicit expression for the conformal Killing tensor. It should be noted that our separation trick, involving the conformally related metric with the Killing tensor, will also work for a wide class of rotating black hole solutions in four and higher dimensions, where the complete separation of variables occurs for massless fields. In particular, it will work for four-charge rotating black holes of four-dimensional string theory [22] as well as for three-charge rotating 
black holes in five-dimensional ungauged $\mathcal{N}=2$ supergravity [23]. We hope to turn to these issues in future works.

\section{ACKNOWLEDGMENT}

The author wishes to thank A. N. Aliev for suggesting this problem and for very helpful discussions. This work is supported by Istanbul Univesity Scientific Research Project (BAP) No. 9227.

[1] M. Demiański and E. T. Newman, Bull. Acad. Polon. Sci., Ser. Sci., Math., Astron., Phys. 14 (1966) 653.

[2] E. T. Newman, L.Tamburino and T. Unti, J. Math. Phys. 4 (1963) 915.

[3] C. W. Misner, J. Math. Phys. 4 (1963) 924; C. W. Misner and A. H. Taub, Zh. Exper. Teor. Fiz. 55 (1968) 223; Sov. Phys. JETP 55 (1969) 122.

[4] W. B. Bonnor, Proc. Cambridge Phil. Soc. 66 (1969) 145.

[5] B. Carter, Commun. Math. Phys. 10 (1968) 280.

[6] D. Bini, C. Cherubini, R. T. Jantzen and B. Mashhoon, Phys. Rev. D 67 (2003) 084013.

[7] D. Bini, C. Cherubini, R. T. Jantzen and B. Mashhoon, Class. Quantum Grav. 20 (2003) 457.

[8] M. Walker and R. Penrose, Commun. Math. Phys. 18 (1970) 265.

[9] E. Radu, Phys. Rev. D 67 (2003) 084030.

[10] A. N. Aliev, Phys. Rev. D 77 (2008) 044038.

[11] C. V. Johnson and R. C. Myers, Phys. Rev. D 50 (1994) 6512.

[12] D. V. Gal'tsov and O. V. Kechkin, Phys. Rev. D 50 (1994) 7394.

[13] N. Alonso-Alberca, P. Meessen and T. Ortin, Class. Quantum Grav. 17 (2000) 2783.

[14] W. Chen, H. Lü and C. N. Pope, Class. Quantum Grav. 23 (2006) 5323.

[15] D. Kubizňàk and V. P. Frolov, Class. Quant. Grav. 24 (2007) F1.

[16] V. P. Frolov and D. Kubizn̆àk, Class. Quant. Grav. 25 (2008) 154005.

[17] A. N. Aliev, H. Cebeci and T. Dereli, Phys. Rev. D 77 (2008) 124022.

[18] G. W. Gibbons and D. L. Wiltshire, Ann. Phys. (N.Y.) 167 (1986) 201; Erratum-ibid. 176 (1987) 393. 
[19] V. P. Frolov and A. Zelnikov and U. Bleyer, Ann. Phys. (Leipzig) 44 (1987) 371.

[20] A. N. Aliev and G. Daylan Esmer, Phys. Rev. D 87 (2013)

[21] Z-W. Chong, G. W. Gibbons, H. Lü and C. N. Pope, Phys. Lett. B 609 (2005) 124.

[22] Z-W. Chong, M. Cvetič, H. Lü and C. N. Pope, Nucl. Phys. B 717 (2005) 246.

[23] Z-W. Chong, M. Cvetič, H. Lü and C. N. Pope, Phys. Lett. B 644 (2007) 192. 